\title{
Observations of a distinctive infra-red spectral feature in the atmospheric spectra of polar stratospheric clouds measured by the CRISTA instrument
}

\author{
Reinhold Spang and John J. Remedios \\ EOS Space Research Center, Department of Physics and Astronomy, University of Leicester, UK
}

Received 28 February 2003; revised 3 June 2003; accepted 10 June 2003; published 30 August 2003.

[1] Polar stratospheric cloud particles (PSCs) are known to strongly influence the infra-red emission spectrum of the lower stratosphere in cold polar winters. The characteristics of these infra-red features have been examined using limb sounding spectra recorded by the CRISTA experiment in August 1997 when many Antarctic PSCs were observed. A distinctive spectral feature centered at $820 \mathrm{~cm}^{-1}$ has been identified in many of these spectra, the first time that a particular band has been observed in the spectra of PSCs in the atmosphere. The feature can be attributed to the $v_{2}$ band of the $\mathrm{NO}_{3}^{-}$ion and strongly suggests a condensed nitric acid component to the particles in the form of solid nitric acid trihydrate (NAT) or liquid ternary solution (STS). The spectral signatures belong to a definite sub-set of the PSC observations recorded by CRISTA. The particles are observed at temperatures well above $192 \mathrm{~K}$, allowing for temperature errors, and it is suggested that NAT particles are the most likely source of the spectral signature. In addition, it is shown that PSC events in which no spectral signature is present are only observed below $192 \mathrm{~K}$ and display a very similar $\mathrm{HNO}_{3}$ temperature relationship to STS particles. INDEX TERMS: 0305 Atmospheric Composition and Structure: Aerosols and particles (0345, 4801); 3360 Meteorology and Atmospheric Dynamics: Remote sensing. Citation: Spang, R., and J. J. Remedios, Observations of a distinctive infra-red spectral feature in the atmospheric spectra of polar stratospheric clouds measured by the CRISTA instrument, Geophys. Res. Lett., 30(16), 1875, doi:10.1029/2003GL017231, 2003.

\section{Introduction}

[2] Infra-red instruments offer considerable potential to determine PSC composition from remote sounding measurements in "local" (balloon instruments), regional (aircraft/satellite) and global (satellite) regimes. This potential had already been exploited by Toon and Tolbert [1995] in suggesting spectroscopic evidence for PSC types other than nitric acid trihydrate (NAT) and ice using solar occultation spectra recorded with a Fourier transform instrument on an aircraft. However, until comparatively recently most infrared detections and analyses of PSCs had been performed with low signal-to-noise data or with data from radiometers measuring the integrated radiance at discrete wavelengths.

[3] More recently, the deployment of new, spectrally resolving, limb sounding instruments has revealed new aspects of PSC spectroscopy in the infra-red. In particular, observations of Arctic PSCs obtained with a balloon-borne instrument (MIPAS-B2) coupled with simulations of the observed spectra have shown that large PSC particles ( $>1 \mu \mathrm{m}$ mean radius) could scatter significant fractions of upwelling tropospheric radiation into limb views [Höpfner et al., 2002]. Höpfner et al. also reported a sharp step observed between 815 and $825 \mathrm{~cm}^{-1}$ and attributed the change in gradient to the $\nu_{2}$ band of the $\mathrm{NO}_{3}^{-}$ion in nitric acid containing PSC particles. Here we report the first observations of a relatively sharp infra-red band as revealed by examination of data from the Cryogenic Spectrometers and Telescopes for the Atmosphere (CRISTA) instrument [Grossmann et al., 2002]. There are two highly valuable aspects to the CRISTA dataset for this purpose: 1) high signal-to-noise for individual spectra, 2) a large number of PSC spectra (>1000) observed in the Antarctic polar winter. In the following sections, we demonstrate the nature of the spectral signature and present evidence for the type of PSC particles observed.

\section{Spectral Observations}

\subsection{PSC Detection From CRISTA Data}

[4] During a space shuttle mission from August 9-15, 1997, CRISTA-2 made observations inside the south polar vortex during mid-winter (coverage up to $74^{\circ} \mathrm{S}$ ). The data represent the first broad band limb emission infra-red spectra of PSCs from satellite instruments. The spectral resolution of the instrument is limited to $2 \mathrm{~cm}^{-1}$, but is adequate for observations of the mostly broad spectral continua which are due to PSCs. Large areas of cloudy regions under different meteorological conditions were detected by CRISTA [Spang et al., 2001]. The enhanced radiation by the clouds produced tremendous intensity changes (Figure 1) with particularly significant changes in the spectral shape, especially at wavenumbers around 800 to $950 \mathrm{~cm}^{-1}$ and 1150 to $1250 \mathrm{~cm}^{-1}$. Parts of these wavenumber regions act as atmospheric windows for clouds and aerosols, because only a few gases with low emissions are present. In Figure 1, examples of spectra for "clear sky" and for optically "thin" and "thick" PSCs are shown. In the thin case, gas emission lines are observed simultaneously with continuum offsets. For optically thick conditions, most of the emission features disappear, the spectrum is similar to a black body spectrum, albeit enhanced over that expected from the local cold temperature, and in addition absorption features occur. The enhanced radiation and absorption features are due to the scattering of tropospheric radiation as noted previously.

[5] Spang et al. [2001] have verified a simple and robust approach for cloud detection by using the ratio of radiances 


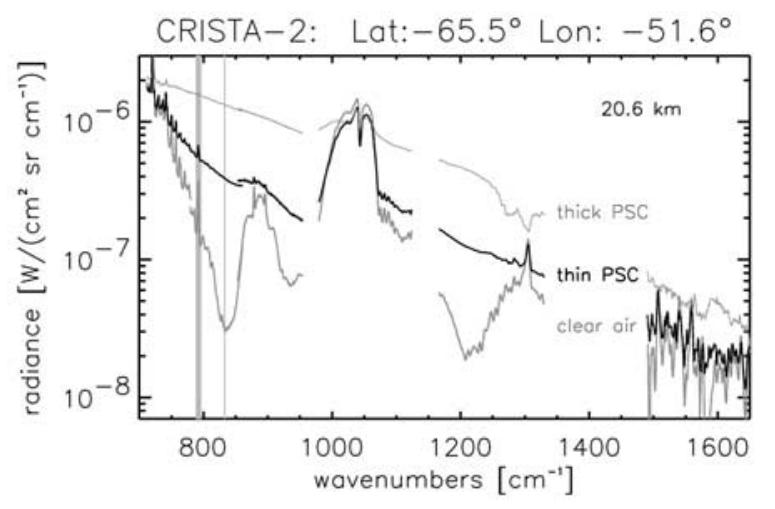

Figure 1. CRISTA-2 PSC spectra, Antarctic vortex, August 1997, 20.6 km: "clear sky", optically thin (lat/long given) and optically thick cases. The grey vertical lines mark the cloud detection wavelength regions.

in the $788-796 \mathrm{~cm}^{-1}$ range, dominated by $\mathrm{CO}_{2}$, and the $832-834 \mathrm{~cm}^{-1}$ regions, dominated by aerosol/cloud emissions (Figure 1, gray vertical lines). The latter region contains only weak contributions from ozone and CFC11 in comparison to the background aerosol and the enhanced cloud emissions. In the following, the defined ratio will be referred to as the standard CRISTA Cloud-Index (CR-CI). The temperature dependence of the ratio is especially weak $(<1 \% / \mathrm{K}$ between 10 and $30 \mathrm{~km})$. Globally, the analyses showed that in the height regime from 12 to $40 \mathrm{~km}$ a ratio of two or less for CR-CI can only be produced by a radiation background anomalously enhanced by aerosols or clouds. However, in the Antarctic polar lower stratosphere, CR-CI < 4.5 indicates the presence of PSC particles. For CR-CI > 1.5 , trace gas concentrations, e.g. for $\mathrm{HNO}_{3}$, can be retrieved from CRISTA data, a slight downward extension of the range used in Spang et al. [2001]. At lower CR-CI values, the optical thickness of the clouds is too great for good retrievals.

\subsection{A Spectral Signature of PSC Type I Particles}

[6] Figure 2 presents a more detailed view of two PSC spectra recorded by CRISTA for the spectral region between $750 \mathrm{~cm}^{-1}$ and $1000 \mathrm{~cm}^{-1}$. The first (diagram (a)) shows a pronounced spectral feature centered at $820 \mathrm{~cm}^{-1}$ which is stronger than the increase in CFC-11 emissions seen at $845 \mathrm{~cm}^{-1}$ in low altitude spectra. This feature is observed in a number of CRISTA spectra of PSCs; PSCs are identified by $\mathrm{CR}-\mathrm{CI}<4.5$, in conjunction with cloud location (Antarctic) and "cloud top height". In contrast, the feature is not observed in the second spectrum shown in diagram (b). The spectra are typical of two classes of detected PSCs that are henceforth referred to as the RE (radiance enhancement) group which show the spectral feature at $820 \mathrm{~cm}^{-1}$ and the NRE (no radiance enhancement) group which do not. The two types are quite different in their radiances near $820 \mathrm{~cm}^{-1}$ but characteristically are very similar in the $830 \mathrm{~cm}^{-1}$ wave number region, which is used for the nominal cloud index. Using a wide range of representative model atmospheres for trace gases, calculations with a radiative transfer model (the Oxford RFM) have shown that the enhancement does not stem from spectral bands of expected stratospheric trace gases. Furthermore, the identified feature at $820 \mathrm{~cm}^{-1}$ is only observed when CR$\mathrm{CI}$ and the tangent altitude of the measurement indicate a PSC is present in the line of sight of CRISTA. Therefore it is certainly a signature of PSC particle formation.

[7] Published refractive indices for PSC particles have also been examined. A number of possible $\mathrm{HNO}_{3}$ containing particles exhibit spectral absorption signatures around $820 \mathrm{~cm}^{-1}$ due to the $\nu_{2}$ band of the $\mathrm{NO}_{3}{ }^{-}$ion (sulphuric ternary solution or STS, binary solutions of $\mathrm{HNO}_{3}$ and $\mathrm{H}_{2} \mathrm{O}$, the meta-stable $\alpha$-NAT, $\beta$-NAT, and nitric acid di- and mono-hydrate abbreviated NAD and NAM respectively). In contrast none of the available refractive indices datasets for water ice show any indications for absorption features in the specified wavelength region. Hence the observed signature is definitely characteristic of PSC type I rather than PSC type II particles.

[8] To further test which PSC type I best fits the observations, we used a forward modeling approach in conjunction with Mie-calculations over a wide range of possible size distribution parameters (lognormal distributions with mean radii from $0.05 \mu \mathrm{m}$ to $10 \mu \mathrm{m}$, width of 1.5 and number densities from 0.3 to $30 \mathrm{~cm}^{-3}$ ). Refractive indices were taken from the datasets of Toon et al. [1994] and Biermann et al. [2000]. The forward modeling accounted for Mie scattering through a calculation of extinction coefficient for the PSC particles but did not explicitly include terrestrial or solar scattering into the line of sight of the instrument. However at $820 \mathrm{~cm}^{-1}$, these effects, as reported by Höpfner et al. [2002], do not show any ability to produce distinct infra-red bands such as the one observed in this study.

[9] From all potential candidates, only $\beta$-NAT and STS with high $\mathrm{HNO}_{3}$ concentration (weight percentages $>40 \%$ ) were able to produce a significant enhancement in the investigated wavelength region with the $\beta$-NAT signature significantly more pronounced than that for STS; $\beta$-NAT is expected to be the most favorable form of NAT in the

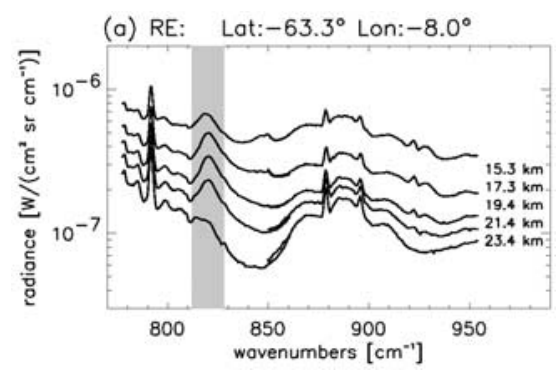

(b) NRE: Lat: $-68.4^{\circ}$ Lon: $-48.3^{\circ}$

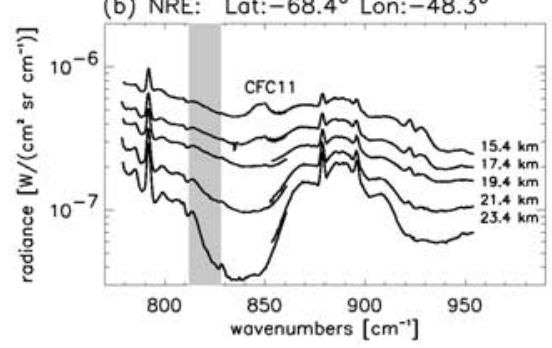

Figure 2. CRISTA-2 PSC limb scans, Antarctic: (a) PSC spectral signature at $820 \mathrm{~cm}^{-1}$ (shaded area); (b) PSC with very flat spectral negative gradient at $820 \mathrm{~cm}^{-1}$. Two detectors are used (overlap at $860 \mathrm{~cm}^{-1}$ ). 
stratosphere [Toon et al., 1994]. Nevertheless, neither STS nor NAT modeled spectra could reproduce the strength of the observed spectral signature (factor 5 and 2.5 respectively smaller enhancements). This disagreement is most likely to be caused by uncertainties in the refractive indices data and needs further investigations. It should also be noted that with increasing mean radii $(\mathrm{r}>1 \mu \mathrm{m})$ the spectral feature at $820 \mathrm{~cm}^{-1}$ becomes progressively broader and more difficult to observe in calculated mie-scattering spectra. Therefore, it is most likely that mean particles sizes for the RE events are of the order of $1 \mu \mathrm{m}$ or smaller.

\section{Statistical Analyses}

\subsection{Definition of an Indicator for PSC Type I Particles}

[10] To quantify the percentage of spectra with enhanced radiation and to determine their correspondence with geophysical factors, an additional radiance ratio (radiance enhancement cloud index or RE-CI) was developed to identify the new PSC spectral feature in a more sensitive way. The ratio, RE-CI, of the mean radiances in the 819 $821 \mathrm{~cm}^{-1}$ range to the strong $\mathrm{CO}_{2}$ emission used in CR-CI ( 788 to $796 \mathrm{~cm}^{-1}$ ) was found to be a simple and effective indicator for the enhancement when employed in combination with CR-CI.

[11] Results for CRISTA data are shown by the two scatter-plots in Figure 3, where the inter-relationships between the two indices are analyzed for the pressure range $25-40 \mathrm{hPa}$ and $40-55 \mathrm{hPa}$. More than 3800 vortex spectra were taken into account (normalized $\mathrm{SPV}>1.6 \cdot 10^{-4} \mathrm{~s}^{-1}$,
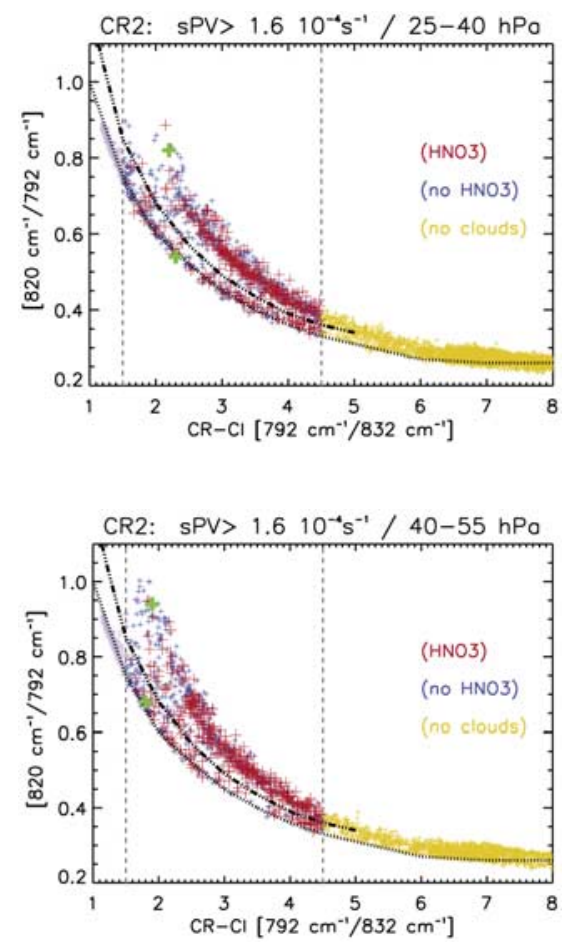

Figure 3. CRISTA cloud indices, CR-CI vs RE-CI, for 25-40 hPa/40-55 hPa: $\mathrm{Red}=\mathrm{PSC} / \mathrm{HNO}_{3}$ data available; Blue = PSC data only; Orange = clear sky; Purple = optically dense PSC; Dash-dotted line = empirical separation line. Dotted line $=$ observed lower limit. Green crosses $=$ CR-CI, RE-CI for spectra of Figure 2.

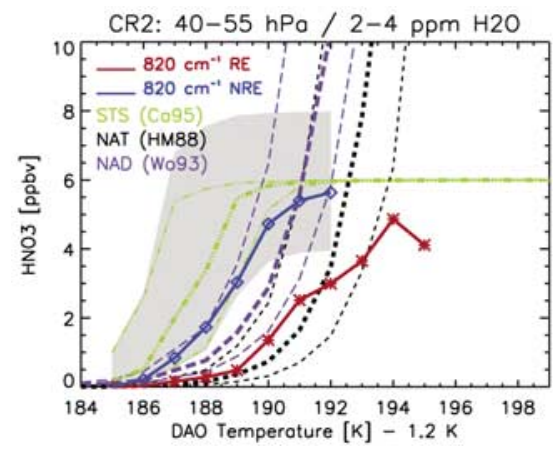

Figure 4. Observed RE (red) and NRE (blue) events (40$55 \mathrm{hPa}$ ) vs. mean CRISTA $\mathrm{HNO}_{3}$ mixing ratios and DAO temperatures ( $1 \mathrm{~K}$ steps at mean pressure). Superimposed are model curves of equilibrium vapor pressures of $\mathrm{HNO}_{3}$ over NAT (black dotted), NAD (purple dashed) and STS (green dash-dotted). Shaded area indicates STS for a range of $\mathrm{H}_{2} \mathrm{O} / \mathrm{HNO}_{3}$ values.

for the definition of sPV see Manney et al. [1994]). Each plot in Figure 3 shows two clear groupings of points, as illustrated by an empirical dividing line (dash-dotted curve). These groupings can be identified as RE above the dividing line and NRE below the dividing line; the identification is illustrated by marking the RE and NRE spectra of Figure 2 on Figure 3. Essentially, for a given CR-CI, RE events will have a larger RE-CI than NRE events as expected from consideration of Figure 2. It is a surprising result that for $1.5<$ $\mathrm{CR}-\mathrm{CI}<4.5$, the RE events $(61 \%)$ comprise a larger fraction of PSC events than NRE events (39\%). Figure 3 also shows that there is a scattering of points, which lie between the bimodal groupings, particularly in the higher pressure range. These points could arise for a number of reasons including views of vertical layers of PSCs of differing characteristics, mixed phase clouds at the tangent point of the measurement and particles of larger mean radius. It was not possible to differentiate these cases in this study.

[12] The separation of the observations into groupings above (RE) and below (NRE) the dividing lines of Figure 3 showed that the RE group of PSCs were observed on average at a temperature of $3 \mathrm{~K}$ higher than the NRE group of PSC events; meteorological analysis temperature data were provided by the GSCF Data Assimilation Office (DAO) [Schubert et al., 1993]. This supports the suggestion of differing particle states as the underlying reason for the spectral signatures observed.

\section{2. $\mathrm{HNO}_{3}$-Temperature Relation}

[13] It is well known that the temperature dependence of the gas phase mixing ratio of $\mathrm{HNO}_{3}$ below approximately $195 \mathrm{~K}$ is related to the composition of PSC particles. Therefore it is interesting to see if the two cloud types in Figure 3 show also a different behavior in their temperature$\mathrm{HNO}_{3}$ relation. As noted previously, only $\mathrm{HNO}_{3}$ retrievals with CR-CI greater than 1.5 are valid. The nature of the CRISTA retrieval scheme for $\mathrm{HNO}_{3}$ also restricted the profile retrievals available to $\sim 40 \%$ of locations of the PSC events in the relevant range here of $1.5<\mathrm{CR}-\mathrm{CI}<$ 4.5(gray dashed lines in Figure 3). This still provides about 400 cases with both PSC spectra and coincident $\mathrm{HNO}_{3}$ 
retrievals for the pressure range $40-55 \mathrm{hPa}$. Cloud-free data are also available at this altitude and analyses show average $\mathrm{HNO}_{3}$ values of around $6 \mathrm{ppbv}$ with a $1-\sigma$ variability of 3 ppbv. Therefore in the equilibrium model calculations for STS presented here, a range of total nitric acid from 4 to $8 \mathrm{ppbv}$ is used to indicate equilibrium limits.

[14] Results are plotted in Figure 4 for the pressure regime between $40 \mathrm{hPa}$ and $55 \mathrm{hPa}$; similar findings result from analysis of the $25-40 \mathrm{hPa}$ level. Model curves for the $\mathrm{HNO}_{3}$-temperature relation are included for equilibrium vapor pressures over NAT [Hanson and Mauersberger, 1988], NAD [Worsnop et al., 1993] and STS [Carslaw et al., 1995]. The curves strongly depend on temperature and on background values of water vapor used in the calculation.

[15] Temperature data have been taken from the DAO analysis fields and adjusted for expected warm biases in the winter polar values; previous analyses of radiosondes have shown a winter polar vortex warm bias of up to $2-3 \mathrm{~K}$ in the analyses depending on height [Spang et al., 2001 and references therein]. The temperature bias is equivalent to a shift for the RE and NRE curves and was taken into account (1.2 $\mathrm{K}$ at $48 \mathrm{hPa}$, the mean pressure for the observations).

[16] Unfortunately coincident water vapor measurements are not available for the CRISTA period. The most appropriate datasets, from POAM III and MLS, show values between 2 and $3.3 \mathrm{ppmv}$ at $40-55 \mathrm{hPa}$ [Nedoluha et al., 2000; Stone et al., 2001]. However, both studies also confirm that there are variations of $\mathrm{H}_{2} \mathrm{O}$ with location in the vortex and altitude due to dehydration effects and descent respectively. Therefore the thermodynamic calculations in Figure 4 have been performed for 2, 3 and 4 ppmv (left-thin, center-thick and right-thin curves respectively). The gray shaded area for STS indicates the potential regime of existence given extreme values for pressure, $\mathrm{H}_{2} \mathrm{O}$, and $\mathrm{HNO}_{3}$.

[17] Figure 4 shows that the $\mathrm{HNO}_{3}$-temperature relation for the RE events is clearly shifted towards higher temperatures compared to that for the NRE events, such that $71 \mathrm{RE}$ events but no NRE events are observed above 192 K. Given the identification of the $\mathrm{NO}_{3}^{-}$band at $820 \mathrm{~cm}^{-1}$, the high temperature existence suggests that the RE particles are NAT. Certainly the $\mathrm{RE} \mathrm{HNO}_{3}$-temperature relationship indicates that the particles exist within the NAT equilibrium regime rather than that for NAD (or STS). However, the gradient of the RE curve does not match well that for the NAT curves. This could be due to the inherent averaging over a range of vortex conditions, the use of equilibrium model calculations, or the potential for RE events to be mixed phase with a major fraction (optically) as RE particles.

[18] In contrast, the NRE events occur only below a clear cut-off at $192 \mathrm{~K}$ and the $\mathrm{NRE} \mathrm{HNO}_{3}$-temperature relationship closely follows equilibrium curves for STS. This suggests that the NRE events for $1.5<\mathrm{CR}-\mathrm{CI}<4.5$ in this time period are mostly STS particles. Again the inherent averaging over the vortex, the assumption of equilibrium models, and the presence of mixed phase clouds may explain differences between the NRE curve and those for STS. However the differences are small and this may also be because mixed phase clouds assigned as NRE events cannot contain significant numbers of RE particles. It should be noted that the NRE curve also falls within the equilibrium existence regime for NAD whilst not matching well the shape of the NAD curves, and that it is also possible for large NAT particles of mean radius greater than $1 \mu \mathrm{m}$ to appear as NRE events.

\section{Conclusions}

[19] Analysis of PSC infra-red emission spectra from CRISTA has enabled the first detection from space of a significant spectral signature at $820 \mathrm{~cm}^{-1}$ in a sub-set of the data. Refractive indices indicate that the feature arises from a nitrate band and the specific candidates appear to be STS and $\beta$-NAT. The observation of the spectral feature at temperatures well above $192 \mathrm{~K}$ indicates that NAT is the most likely explanation for these events. In addition, cloudy events without the spectral signature are formed only below $192 \mathrm{~K}$ and display a similar $\mathrm{HNO}_{3}$-temperature relationship to that for STS. Unfortunately the $820 \mathrm{~cm}^{-1}$ band cannot be reproduced by existing refractive indices and new laboratory measurements for candidate species are a priority.

[20] Acknowledgments. We would like to thank D. Offermann for encouragement, S. Massie and A. Dudhia for Mie-calculation and radiative transfer codes respectively, G. Nedoluha for POAM III data, the reviewers and also the EU for support (FP 5, MAPSCORE, EVK2-CT-2000-00077).

\section{References}

Biermann, U. M., et al., Absorption spectra and optical constants of binary and ternary solutions of $\mathrm{H}_{2} \mathrm{SO}_{4}, \mathrm{HNO}_{3}$ and $\mathrm{H}_{2} \mathrm{O}$ in the mid infrared at atmospheric temperatures, J. Phys. Chem. A, 104, 783-793, 2000

Carslaw, K. S., et al., An analytic expression for the composition of aqueous $\mathrm{HNO}_{3}-\mathrm{H}_{2} \mathrm{SO}_{4}$ stratospheric aerosols including gas phase removal of $\mathrm{HNO}_{3}$, Geophys. Res. Lett., 22, 1877-1880, 1995.

Grossmann, K. U., et al., The CRISTA-2 Mission, J. Geophys. Res., 107(D23), 8173, doi:10.1029/2001JD000667, 2002.

Hanson, D., and K. Mauersberger, Laboratory studies of the nitric acid trihydrate: Implications for the Antarctic ozone hole, Geophys. Res. Lett., 15, 855-858, 1988 .

Höpfner, M., et al., Evidence of scattering of tropospheric radiation by PSCs in mid-IR emission spectra: MIPAS-B observations and KOPRA simulations, Geophys. Res. Lett., 29(8), 1278, doi:10.1029/2001GL014443, 2002.

Manney, G. L., et al., On the motion of air through the stratospheric polar vortex, J. Atm. Sci., 51, 2973-2994, 1994.

Nedoluha, G. E., et al., POAM III measurements of dehydration in the Antarctic lower stratosphere, Geophys. Res. Lett., 27, 1683-1686, 2000.

Schubert, S. D., et al., An assimilated dataset for Earth Science Applications, Bull. American Meteorol. Soc., 74, 2331-2342, 1993.

Spang, R., et al., CRISTA-2 observations of the south polar vortex in winter 1997: A new dataset for polar process studies, Geophys. Res. Lett., 28, 3159-3162, 2001

Stone, E. M., et al., The onset, extent and duration of dehydration in the Southern Hemisphere polar vortex, J. Geophys. Res., 106, 22,97922,989, 16 Oct., 2001.

Toon, O. B., and M. A. Tolbert, Spectroscopic evidence against nitric acid trihydrate in polar stratospheric clouds, Nature, 365, 218-221, 1995.

Toon, O. B., et al., Infrared optical constants of $\mathrm{H}_{2} \mathrm{O}$ ice, amorphous nitric acid solutions, and nitric acid hydrates, J. Geophys. Res., 99, 21,63125,654, 1994 .

Worsnop, D. R., et al., Vapor pressures of solid hydrates of nitric acid: Implications of polar stratospheric clouds, Science, 259, 71-74, 1993.

J. Remedios and R. Spang, EOS, Space Research Center, Dept. of Physics and Astronomy, Univ. of Leicester, Univ. Road, Leicester, LE1 7RH, UK. (R.spang@le.ac.uk; j.j.remedios@le.ac.uk.) 